\title{
The effect of spatial order on piecemeal shape recognition: A developmental study
}

\author{
JOAN S. GIRGUS \\ Princeton University, Princeton, New Jersey 08540 \\ LEON H. GELLMAN \\ Sarah Lawrence College, Bronxville, New York 10708 \\ and \\ JULIAN HOCHBERG \\ Columbia University, New York, New York 10027
}

\begin{abstract}
Stationary shapes were displayed piecemeal by moving an aperture from one place to another around their contours. Shape recognition was poorer with an unpredictable order of display. This result is more consistent with hypothesis-testing than with either eye-movement or visual information models of shape recognition. Shape recognition was poorer for 9- and 11 year-old children than for adults.
\end{abstract}

If an entire shape or scene is discernible in a single glance, it is not necessary to postulate that either eye movements or information storage is necessary for the perception of that shape or scene to occur. However, when the shape or scene is too large to be clearly visible in a single glance, the viewer must direct his gaze successively at different details of the object or scene in order to see them with sufficient clarity. Under these circumstances, the viewer must be able to keep track of where the successive glimpses fall relative to one another, as well as of what falls where within each glimpse. Since many shapes and most scenes subtend a visual angle that is too large to permit the shape or scene to be seen clearly in a single glimpse, most of the visual information that we pick up about the shapes of objects and how they are laid out to create scenes is normally obtained by successive discontinuous glimpses. This fact raises the following problem which is fundamental to any understanding of visual perception: How can we tell from two successive, momentary glimpses of parts of an object or scene where those parts lie on that object or in that scene?

One possible answer to this question is that we know which way our eyes have moved with each glance and, by using that knowledge, can assign the information in each separate glimpse to its proper

Parts of this study were supported by NICHHD Grant HD06002-02 to the first author while she was at The City College of the City University of New York and by NICHHD Grant 5 RO1 HD 06768.02 and NIE Grant G 740099 Rev 2 to the last author. Requests for reprints should be sent to Joan $S$. Girgus, Department of Psychology, Princeton University, Princeton, New Jersey 08540. place in the visual environment. Provided that we can retain the information about the content and location of the first glimpse until the last informative glance has occurred, the shape would be as available to perception as if all of its parts had been simultaneously clearly visible.

According to such an explanation, the viewer needs only the normal information available to him about the direction of his successive eye movements in order to assign the details each glance provides to their appropriate place in the overall shape. For information about successive gaze directions, we can draw on either or both of two general approaches to the perception of movement and stability. Efference record theories assume that the visual system keeps track of the direction and extent that the eye has been ordered to move (Festinger, Ono, Burnham, \& Bamber, 1967; Matin, 1972). Formally similar for this purpose is the proposal (Gibson, 1966) that the transformations between the overlapping retinal images that occur when the eye changes its point of view contain within them enough purely visual information to specify the shape of an object or the stable layout of a scene; for example, Johansson's vector-analysis model of movement perception (1950) suggests that the common vectors of retinal translation would provide the framework within which the successive views take their places. Either or both of these approaches would account for how we assign the successive glimpses of the parts of an object or scene to their correct places in space and thus to their correct relative (configurational) loci. It should be noted that neither of these explanations places any restriction on the temporal order in which the frag- 
mentary views are received, as long as the viewer is given adequate information about the relative directions of each successive view.

Alternatively, it has been argued (Hebb, 1949; Hochberg, 1968; Neisser, 1967) that shape perception requires more than the perception of relative location in space: It requires the perceiver to match the information contained within one or more glimpses with a stored "map" or anticipatory schema. In such theories of shape perception (which may, indeed, assume that eye movements are essential in the initial formation of a schema; cf. Hebb, 1949), the schematic map generates visual expectations about what will be found at some place on an object or in a scene. The eye movements that generate the successive glimpses are normally executed to answer some perceptual question, so that information about where the eye is pointed may contribute to, but be neither necessary nor sufficient for, the perception of shapes that are glimpsed piecemeal. On the other hand, it should be relatively easy to make temporal order important to any process that is based on expectancy testing.

Evidence that eye movements, and efferent information about them, are not necessary for the recognition of a shape that is presented in successive fragments has been provided by experiments in which an object is systematically and continuously moved around behind a small stationary aperture, through which only one piece of the contour is visible at a time [Girgus, 1973, 1976; Girgus \& Hochberg, 1970; Hochberg, 1968; Murphy, 1973). Under appropriate circumstances, the shape of the object is readily perceived by adults even though the viewer's eye remains approximately stationary in this situation. (It should be noted that even if eye movements should occur and the successive glimpses that the viewer obtains through the stationary aperture should fall on different retinal locations, the successive entries should be correctly perceived as occurring at the same place in space because of the normal compensation for eye movements.) Thus, neither eye movements nor their compensation mechanisms can provide any useful information about the stationary aperture-viewing situation from the standpoint of efferent record theories, and we must look elsewhere for an explanation of how shapes are recognized in this situation. On the other hand, visual information theories can explain the fact that the perception of shaye occurs in the stationary aperture-viewing situation, since visual position information from the successive glimpses through the aperture will continue to be available as long as there is either continuous movement or overlap between views, independent of whether any eye movements have occurred. The present experiment was intended to show that neither efferent nor visual information about where the eye is voluntarily directed is necessarily sufficient to achieve shape recognition under conditions of successive presentations of parts of a shape. In this experiment, an aperture moved over a stationary shape, so that each fragment of the shape that was seen through the aperture was presented at its appropriate location in space relative to the other fragments. By the end of each series of such presentations, the entire shape had been presented as the locus of points at which the aperture had been shown. The piecemeal views of each shape were ordered in two different ways. In the random condition, parts of the contour of the stationary object were briefly revealed through an aperture that appeared at unpredictable places on the object. In the systematic condition, the aperture was moved contiguously around the contour of the object.

In both conditions, the shane was specified by the locus of places at which the aperture was presented, so that there was no real need for the viewer to resolve the detail within the aperture in order to discern the shape. Throughout both conditions, the visual field contained a strong stationary visible framework within which the aperture was successively displayed so that no autokinetic effects were present. In neither the random nor the systematic condition was the viewer ever called upon to make saccades greater than $5 \mathrm{deg} 30 \mathrm{~min}$, so that it is virtually certain that successive glances provided $100 \%$ overlap between views in both conditions, as far as the field occupied by the underlying shape is concerned.

Neither an efferent record theory nor a visual information theory of eye-movement compensation would predict any differences in shape recognition to occur under these two conditions. If the eye remains stationary during the presentation, the locus of places in which the aperture appeared will have outlined the shape on the retina by the end of each presentation sequence in both the random and the systematic conditions. If the eye fixates the successive presentations of the moving aperture, the visual and nonvisual information that is normally expected to accompany voluntary saccades should allow the aperture to be assigned its correct location in space in both conditions.

There are, of course, other differences between the two conditions. In the systematic condition, the overlap between successive views provides a longer uninterrupted view of any part of the shape, whereas in the random condition, more repeated samples of each region are provided. In the systematic condition, the path of the aperture's movement follows the outline of the shape, whereas in the random condition, the endpoint of the movements of the aperture lie on the shape's outline but the directions of the individual movements are unrelated to the shape.

As long as the two conditions present each part of the shape for the same total time, however, and each view in the random condition is shown for a long 
enough time for its location in the stationary framework and the visual field to be registered by the viewer, neither efferent record theories nor visual information theories, as they stand, predict a difference in the shape's recognizability. It is true that Gibson's explanation calls for some reasonable, but as yet unspecified, amount of overlap between successive views. The overlap required by this explanation is, however, needed to provide information about the relative locations of the aperture in successive views. As noted above, substantial overlap of this sort is present in both the random and the systematic conditions. It should be noted that all of the theories mentioned consider perception to involve active exploration. The function of the activity in Gibson's theory, however, is to provide information about the invariant spatial layout of the stimulus which, as we have noted, is provided in both conditions of this experiment. On the other hand, because the randomly revealed views would not, in general, provide answers to the viewer's own perceptual inquiries, theories that posit such perceptual testing (Hochberg, 1968; Neisser, 1967) would predict that shape recognition in this condition would be inferior to that in the condition in which the views were systematically ordered.

This study was also designed to explore whether any effect of scanning order that may be obtained for children of 9 and 11 years of age is significantly different from that obtained for adults. Previous studies have found that the ability to recognize simple, familiar shapes under conditions of piecemeal presentation decreases as the age of the subject decreases (Girgus, 1973, 1976; Girgus \& Hochberg, 1970, 1972), but the basis for the developmental relationship is not known. It is possible that increasing mastery of schematesting skills with age might account for the fact that the recognition of simple, familiar shapes improves with age in the aperture-viewing situation, although, of course, other mediating processes might underlie this improvement. If random and systematic orders of presentation of the pieces of a shape make different demands on the viewer's schema-testing abilities, we might expect different developmental trends for the two conditions. The ages used were chosen because they had shown substantial differences in the previous experiments on piecemeal shape recognition and because they were the youngest ages at which the response task used in this experiment could readily be performed.

\section{EXPERIMENT 1}

\section{Method}

Subjects. There were 12 subjects in each of three age groups, with mean ages of 8 years 10 months, 10 years 8 months, and 22 years, respectively. The younger two groups of subjects were chosen by age from a public school in New York City; the oldest group of subjects was composed of paid volunteers from a public college in New York City.

Stimuli. Figure 1 shows the square, the cross, and the block " $E$ " that were used as the stimuli in this experiment. The sides of the square and the cross were all the same length and were the same length as the shortest sides of the block "E."

In order to control the order in which the various parts of the shape were viewed, motion pictures were made in which a circular aperture was moved over the contour of the shape. Since the diameter of the aperture was equal in size to one side of the square, only that amount of the shape was visible in any one view through the aperture. Projected on a screen placed 24 in. in front of the subject, the aperture subtended a visual angle of $1 \mathrm{deg} 29 \mathrm{~min}$.

In the systematic condition, the aperture was moved around over the contour of the shape in either a clockwise or a counterclockwise direction. Frame-by-frame filming was used to produce motion pictures in which each view was one-eighth of the length of one side of the square farther along the contour than the previous view. Thus, there was considerable overlap between the contents of the aperture in successive views. This procedure provides motion pictures of a smoothly moving aperture, systematically circumnavigating the contour of a shape.

In the random condition, the aperture was moved randomly from place to place on the contour of the shape. The order of the views for this condition was determined by randomly ordering the views from the systematic films. In these films, the aperture appeared to jump unsystematically around the contour of the shape.

The set of views that was presented was identical in the two conditions, so that the subject was shown the entire contour of each shape under both conditions. In both conditions, furthermore, each corresponding glimpse of the contour was presented at the same location within a stationary gray rectangular framework which was provided by the boundary of the motion picture projection on the projection screen and which subtended visual angles of $13 \mathrm{deg} 38 \mathrm{~min} \times 10 \mathrm{deg} 16 \mathrm{~min}$. In other words, the appropriate part of the stationary shape was revealed in each glimpse through the aperture in both conditions.

Although the primary difference between the two kinds of motion pictures was the order and, hence, the degree of overlap with which the apertures were presented, it should be noted that different kinds of eye movements may have been encouraged
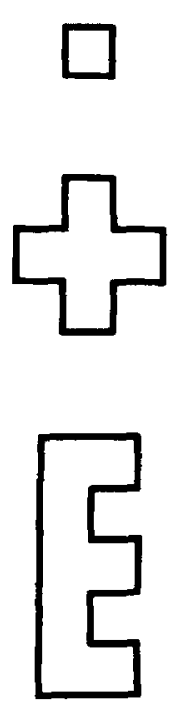

Figure 1. The square, the cross, and the block " $E$ " used as stimuli in this experiment. 
by the two procedures. One might expect the smoothly, systematically moving aperture would elicit smooth-tracking eye movements, while the randomly jumping aperture would elicit saccades. The motion pictures were presented at the rate of $500 \mathrm{msec}$ per view in both conditions, therefore, in order to provide sufficient time for saccades to occur.

Procedure. Each potential subject was first given a pretest with two right-angled shapes (a block " $L$ " and a block " $H$ ") that were different from the test shapes. Each potential subject was given two oppo:tunities to look at each of the pretest shapes for approximately $5 \mathrm{sec}$ and then draw that shape from memory. Only subjects who drew both pretest shapes correctly were shown the motion pictures of the test stimuli. The pretest was used to ensure that all subjects used in the actual experimental conditions could draw shapes similar to the test shapes when the shapes were no longer in view.

Each subject then received each of the three test shapes under both systematic and random conditions. Within each age group, one of the two sets of films was presented first to one half of the subjects and the other was presented first to the other half of the subjects, and the three test shapes were presented an equal number of times in every possible order.

Before viewing each of the two sets of films, the subjects were shown mechanical demonstrations of an aperture moving around over the contour of each of the pretest shapes in a manner appropriate to the films that they were about to see. The subjects were told that they were going to see movies that looked like the mechanical demonstration, that the pictures in the movies would be different from the pictures they had seen in the pretest, and that after each movie they would be asked to draw the picture that appeared under the hole in the movie.

A subject could be shown any one test film from one to four times. After each presentation of the film, the subject was asked to draw the shape that he had just seen in the movie. If he drew the shape correctly, the number of times he had seen that film was recorded and a new test film was presented. If, after four consecutive presentations of a test film, the subject still failed to draw the shape correctly, a score of 5 presentations was assigned for that test shape in that condition and a new test film was presented. Thus the score for any subject on each test shape and input condition could range from 1 to 5 presentations; the lower the score, the better the subject's performance.

It should be noted that a drawing was scored as correct if the parts of the shape were present in their correct relationships, without regard to the correctness of the relative sizes of the various stimulus dimensions. The responses were not difficult to score; the drawings either were clearly recognizable as the correct shape or were clearly some other shape (or no recognizable shape at all); the subjects did not draw partially or schematically correct shapes, probably because the stimulus shapes were both simple and familiar to the subjects.

\section{Results}

Table 1 shows the mean number of trials needed for a correct response per age group, movement condition, and test shape. An analysis of variance performed on these data indicates that all three main

Table 1

Mean Number of Trials to Correct Response per Age Group, Movement Condition, and Test Shape

\begin{tabular}{|c|c|c|c|c|c|c|c|}
\hline \multirow{2}{*}{$\begin{array}{l}\text { Mean } \\
\text { Age* }\end{array}$} & \multirow[b]{2}{*}{$\mathbf{N}$} & \multicolumn{3}{|c|}{ Random Movement } & \multicolumn{3}{|c|}{ Systematic Movement } \\
\hline & & Square & Cross & $\mathbf{E}$ & Square & Cross & $\mathbf{E}$ \\
\hline 8.8 & 12 & 2.33 & 4.33 & 4.42 & 1.67 & 3.50 & 4.58 \\
\hline 10.7 & 12 & 2.58 & 3.91 & 4.08 & 1.92 & 2.83 & 3.83 \\
\hline 22.0 & 12 & 1.33 & 3.83 & 3.08 & 1.50 & 1.42 & 1.75 \\
\hline
\end{tabular}

*In years. effects showed significant differences with $\mathrm{p}<.01$ [age, $\mathrm{F}(2,30)=9.47$; movement condition, $\mathrm{F}(1,30)=$ 22.32; shape, $F(2,60)=29.28)$. The means in Table 1 indicate that the younger the subjects, the more difficulty they will have in processing shapes under the conditions used in this experiment. It should be noted, however, that only the differences between the oldest and the youngest age groups was significant, with $\mathrm{p}<.05$, when a Tukey (B) test was performed on these data. The means in Table 1 seem to indicate further that the square was easier to identify than either the cross or the block $E$, while the cross and the block $E$ were about equally difficult to identify. The appropriate Tukey (B) tests statistically confirmed this description. Table 1 also clearly shows that it was considerably more difficult for subjects to process the shape information when the aperture jumped randomly around the contour than when it moved smoothly and systematically around the contour.

Only one of the possible interactions in this design was significant; this was the interaction between movement condition and test shape, which was significant at the .05 level $[\mathrm{F}(2,60)=4.08]$. Tukey (B) tests performed on these data revealed that the cross was significantly more affected by the differences between the two movement conditions than either the square or the block $\mathrm{E}$.

In order to see whether there was any tendency for performance on this task to improve with practice, a two-way analysis of variance was performed, using age and trials as the variables. As expected, the main effect of age was significant $[F(2,30)=9.47, p<.01]$. However, neither the main effect of trials nor the interaction between age and trials was significant [trials, $F(5,120)=.71$; Age by Trials, $F(5,120)=.62$ ]

\section{Discussion}

The effects of age and test shape on the ease of shape processing replicate several earlier studies concerned with shape processing under aperture-viewing conditions (Girgus, 1973, 1976; Girgus \& Hochberg, $1970,1972)$. The ability to process sequential shape information efficiently and accurately seems to improve with age and to be sensitive to a number of stimulus parameters. One of these may be the amount of information contained in the shape, which, as Attneave (1954) has suggested, may be roughly measured by the number of corners. The significant interaction between test shape and movement condition may imply that the processing of symmetrical shapes is particularly affected by random information input. The absence of any significant interaction between age and method of presentation, however, means that we have no reason to attribute children's poor performance at piecemeal shape recognition to deficiencies in their schema-testing abilities (or for that matter to the deficiencies that have been observed in their scanning abilities; cf. Vurpillot, 1968).

It is not clear how any explanation of successive 
piecemeal shape perception in terms of assigned locations in space, whether based on the visual information produced by the optical transformations provided by overlapping successive views or by an efference record theory, would explain the fact that the random-movement condition was more difficult than the systematic-movement condition. Both conditions provide sufficient information about the layout of all of the parts of the shapes for them to be recognized, inasmuch as the view of the shape that was displayed at each position of the aperture during the systematic sampling was also provided when the apperture appeared in the corresponding position during the random presentation condition, and inasmuch as the loci of the apertures themselves fully specified each shape.

It is possible, however, that the $500 \mathrm{msec}$ per view that was provided was not enough time for the execution of the eye movements required by the random presentation condition and for the processing of the information about the aperture's location provided by the eye-movement compensation mechanisms under those time constraints. It is conceivable, for example, that, when subjects try to fixate the aperture at an unexpected place in each of a long succession of views and to assimilate the information that each contains, $500 \mathrm{msec}$ is insufficient to register each new location of the aperture in peripheral vision and to initiate and execute an accurate fixation movement or to compensate for that movement once it is executed. While it seemed unlikely, given what is known about eye-movement latencies, that the presentation rate was too fast to permit accurate registration and fixation of the locus of positions occupied by the aperture, it is possible to explore this possibility by eye-movement measurement. Thus, we undertook the following supplementary experiment to test this possibility, and to provide pilot data for future research if the conditions in this experiment, in fact, elicited unusual and inadequate looking behavior.

\section{EXPERIMENT 2}

\section{Method}

Subjects. The subjects were one adult ( 21 years) and two children ( 10 years 4 months and 8 years 1 month).

Stimuli. The stimuli were the films of the square and the cross

Procedure. Each subject viewed the four films in the following order: systematic cross, systematic squarc, random cross, and random square. The pretest and training procedures were identical to those described previously. However, a verbal report was used rather than a drawing task because the eye-movementrecording conditions made drawing impossible.

Each subject's eye-movement patterns were recorded by means of a Whittaker Series 1900 Eye View Monitor and T. B. Pupilometer system, while the subject was positioned in a headrest (see Young \& Sheena, 1975). Under these conditions, the system is relatively free from error due to small head movements and its accuracy is better than 1 deg 30 min of visual angle for both horizontal and vertical eye movements.

\section{Results and Discussion}

In scoring the eye-movement data, the $500 \mathrm{msec}$ during which each view was present in the aperture was broken up into two 250 -msec segments. Each 250-msec segment was sampled twice, photographically, for durations of $44 \mathrm{msec}$ separated by $81 \mathrm{msec}$. Every time a subject's eye was fixated on the aperture during an entire interval of $169 \mathrm{msec}(44+81+44)$, it was scored as a "hit." Table 2 shows the proportion of "hits" achieved by each subject for each stimulus during each of the two 250 -msec time periods that each view was visible. As can be seen from this table, the proportion of "hits" during the first 250 msec segment ranged from .71 to .99 for both of the systematic stimuli, but only from .29 to .63 for the two random stimuli. However, this difference between the random and systematic stimuli disappeared during the second 250-msec time period that each was seen. During this time period, the proportion of "hits" for the systematic stimuli ranged from .67 to .99 , while the proportion of "hits" for the random stimuli ranged from .78 to .95 .

Note that Table 2 shows no sign of age differences in the proportion of "hits" for either of the 250msec segments per view or in the proportion of "hits" found for the two stimuli in either viewing segment.

Thus, although it may take longer for subject to fixate the aperture under random movement than under systematic movement conditions, the $500-\mathrm{msec}$ viewing time available for each view in this experiment makes it extremely likely that subjects of all ages could register the direction of the aperture during each 500-msec presentation in the random condition. In fact, they probably had at least 169 to $250 \mathrm{msec}$ per view during which their eyes were fixated on the aperture under both random and systematic conditions. It should be noted that, with longer effective

Table 2

Proportion of Fixations ("Hits") Achieved by Each Subject During Each of the 250-Msec Viewing Segments Available per View

First 250 Msec

\begin{tabular}{cccccc}
$\begin{array}{c}\text { Mean Age } \\
\text { in Years }\end{array}$ & \multicolumn{2}{c}{ Systematic Condition } & & \multicolumn{2}{c}{ Random Condition } \\
\cline { 2 - 3 } Square & Cross & & Square & Cross \\
\hline 8.1 & .71 & .80 & .30 & .38 \\
10.3 & .91 & .90 & .63 & .29 \\
21.0 & .80 & .99 & .38 & .29 \\
\hline
\end{tabular}

Second $250 \mathrm{Msec}$

\begin{tabular}{|c|c|c|c|}
\hline \multicolumn{2}{|c|}{ Systematic Condition } & \multicolumn{2}{|c|}{ Random Condition } \\
\hline Square & Cross & Square & Cross \\
\hline 67 & .79 & .81 & .90 \\
\hline .97 & .87 & .91 & .95 \\
\hline .86 & .99 & .89 & .78 \\
\hline
\end{tabular}


viewing times in the systematic than in the random condition, the former may provide more opportunity for processing and encoding, although the stimulus information is equivalent in both conditions.

\section{CONCLUSIONS}

Earlier experiments in aperture-viewing showed that eye movements were not necessary for the recognition of shapes viewed by successive partial presentations through an aperture. The present data show that eye movements are not sufficient for shape' perception under aperture-viewing conditions. On the other hand, if the subjects in this experiment were trying to anticipate what would be seen in each successive glimpse, they could far more readily form and test hypotheses about what they would see next in the systematic condition, in which the aperture moved in a predictable path around the contour of the shape, than they could in the random condition, in which such anticipation was not possible. Thus, the data from this experiment are consistent with the view (Hochberg, 1968, 1972; Neisser, 1967) that shape perception involves a kind of hypothesistesting procedure, with the hypotheses generated from stored "maps" or anticipatory schemas. At most, however, experiments like these can only be used to argue with more or less plausibility that the viewer is able to draw upon schematic maps when trying to recognize shapes that are presented piecemeal. Other methods will be required to demonstrate whether this ability is also used in the perception of shape under more normal circumstances.

\section{REFERENCES}

Attneave, F. Some informational aspects of visual perception. Psychological Review, 1954, 61, 183-193.
Festinger, L., Ono, H., Burnham, C., \& Bamber, D. Efference and the conscious experience of perception. Journal of Experimental Psychology, Monograph Supplement, 1967, 74(4, Whole No. 637).

Gibson, J. J. The senses considered as perceptual systems. Boston: Houghton Mifflin, 1966.

Girgus, J. S. A developmental approach to the study of shape processing. Journal of Experimental Child Psychology, 1973, 16, 363-374.

Girgus, J. S. A developmental study of the effect of eye movements in the processing of shape information. Journal of Experimental Child Psychology, 1976, 22, 386-399.

Girgus, J. S., \& Hochberg, J. Age differences in sequential form recognition. Psychonomic Science, 1970, 21, 211-212.

Girgus, J. S., \& Hochberg, J. E. Age differences in shape recognition through an aperture in a free-viewing situation. Psychonomic Science, 1972, 28, 237-238.

He BB, D. The organization of behavior. New York: Wiley, 1949.

HochberG, J. In the mind's eye. In R. Haber (Ed.), Contemporary theory and research in visual perception. New York: Holt, Rinehart, \& Winston, 1968.

Hochberg, J. Perception, I. Color and shape. In J. W. Kling \& L. A. Riggs (Eds.), Woodworth \& Schlosberg's experimental psychology (3rd ed.). New York: Holt, Rinehart \& Winston, 1972.

Johansson, G. Configurations in event perception. Uppsala, Sweden: Almquist and Wiksell, 1950.

Matin, L. Eye movements and perceived visual direction. In D. Jameson \& L. Hurvich (Eds.), Handbook of sensory physiology (Vol. 7, Pt. 4). Berlin: Springer-Verlag, 1972.

Murphy, R. Recognition memory for sequentially presented pictorial and verbal spatial information. Journal of Experimental Psychology, 1973, 11, 327-334.

Neisser, U. Cognitive psychology. New York: Appleton, 1967.

VuRPILLOT, E. The development of scanning strategies and their relation to visual differentiation. Journal of Experimental Child Psychology, 1968, 6, 632-650.

YounG, L. R., \& Sheena, D. Survey of eye movement recording methods. Behavior Research Methods \& Instrumentation, 1975, 7, 397-429.

(Received for publication August 21, 1979; revision accepted May 7, 1980.) 